\title{
EXTENDED FREQUENCY COMPENSATION OF A DIAMAGNETIC LOOP
}

\author{
J. H. BooskE* W. D. GetTY† and R. M. GilgeNBach* \\ * Department of Nuclear Engineering, University of Michigan. Ann Arbor. Michigan 48109. L.S.A. \\ + Department of Electrical Engineering and Computer Science. University of Michigan, Ann Arbor. \\ Michigan 48109, U.S.A.
}

(Received 4 March 1986)

\begin{abstract}
A method of compensation for a diamagnetic loop that is magnetically coupled to a concentric stainless-steel vacuum vessel is presented. This compensation method accounts for imperfect magnetic coupling between the vessel eddy currents, the diamagnetic loop. and the plasma diamagnetic currents. and it also corrects for a finite loading resistance on the diamagnetic loop. A procedure for adjusting and calibrating the active-filter compensation circuit is presented. It can be applied to internal or external diamagnetic loops.
\end{abstract}

\section{INTRODUCTION}

THE VALUE of diamagnetic loops for diagnosis of hot plasmas is well known. Diamagnetic loops give a direct measurement of average plasma energy density that. in combination with a particle density measurement, gives a measure of average particle kinetic energy. In many experiments diamagnetism is measured by loops that encircle a long, thin plasma column. The loop may be placed inside or outside of the vacuum vessel. and the signal is usually processed by passive or active analog integration in the time domain, or an equivalent digital data-processing procedure. If the vessel is metal, one must make corrections for eddy currents induced in the walls by plasma currents. If the vessel is made of relatively low-conductivity metal and the vessel radius is not much larger than the plasma axial length. an external diamagnetic loop can be effective diagnostic. The need for a vacuum feedthrough is eliminated and the user has ready access to the loop. The penalty in using an external loop is that the high-frequency response is limited by the magnetic-flux diffusion times of various wall-current modes representing higher-order. nonuniform current distributions in the vessel wall. If the higher order diffusion time constants are small compared to the dynamic plasma time scales of interest. the higher-order diffusion process can be ignored and the current distribution in the wall can be assumed to be uniform, leading to an $R-L$ equivalent circuit model for the vessel which is mutually coupled to the plasma. If an internal loop is used. high-frequency response is improved but one must still consider zero-order mutual coupling between the plasma, vessel wall and diamagnetic loop.

The purpose of this paper is to demonstrate how a diamagnetic loop can be compensated for low-frequency wall coupling and. additionally. for two non-ideal effects. These effects are imperfect magnetic coupling (i.e. mutual coupling coefficients between coupled inductors of less than unity) between the plasma currents. the vessel wall, and the diamagnetic loop. and finite resistive loading of the diamagnetic loop by the signal-processing circuit. The general method can be applied to either internal or external loops, but is presented here in terms of an external loop. In previous related work. RothmaN (1968) has considered vessel eddy currents but assumed 
perfect magnetic coupling and zero loading of the diamagnetic loop. He gave the basic active integrator circuit that can be used for compensation under these approximations. BOTTIGLIONI (1970) has done a detailed analysis of the effect of finite magnetic flux diffusion time through the vessel wall for both internal and external loops, and showed that the high-frequency response of an external loop is limited by the diffusion times of the higher-order wall current modes, and the low-frequency response is governed by the decay time of the zero-order mode, uniformly distributed wall current. The present model accounts for vessel eddy currents on the uniformcurrent flux diffusion time scale, imperfect magnetic coupling, and diamagnetic loop loading. The analysis provides a method for the design of an active analog filter for signal processing, and an experimental method of calibration and frequency compensation for diamagnetic loops. The nonuniform-current flux diffusion time is not considered. For typical stainless-steel vacuum vessels the uniform-current flux diffusion time is of the order of $60-70 \mu \mathrm{s}$, and the nonuniform-current time is of the order of $1 \mu \mathrm{s}$.

\section{MODEL}

In the present approximation, the diamagnetic loop, vessel and plasma can be modeled as lumped, coupled inductors as shown in Fig. 1. The flux linkages of these inductors are given by
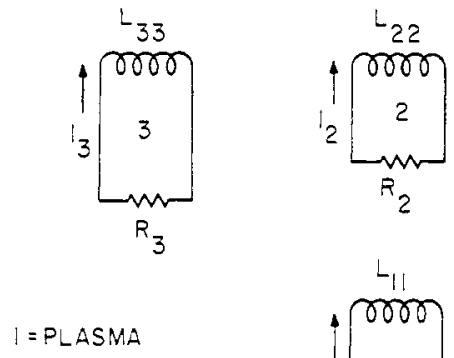

2 = VACUUM VESSEL

$3=$ DIAMAGNETIC LOOP

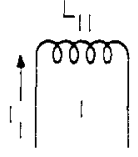

FIG. 1.-Linked inductor model used to design the diamagnetic loop compensation circuit.

$$
i_{m}=\sum_{n=1}^{3} L_{m n} \cdot i_{n}, \quad m=1,2,3
$$

where the inductance matrix is given by

$$
[L]=\left[\begin{array}{lll}
L_{11} & M_{12} & M_{13} \\
M_{21} & L_{22} & M_{23} \\
M_{31} & M_{32} & L_{33}
\end{array}\right] .
$$

The correspondence between the inductances and the plasma, vessel and loop are defined in Fig. 1. It is assumed that $[L]$ is symmetric. 
The external circuit constraints on the vessel equivalent circuit and the diamagneticloop equivalent circuit are given by

$$
\begin{aligned}
& v_{2}(t)=\mathrm{d} i_{2} / \mathrm{d} t=-R_{2} \cdot i_{2} \\
& v_{3}(t)=\mathrm{d} i_{3} / \mathrm{d} t=-R_{3} \cdot i_{3}
\end{aligned}
$$

where $R_{2}$ is the equivalent resistance of the vessel wall and $R_{3}$ is the diamagneticloop loading resistance. The "plasma current" $i_{1}$ is assumed to be a known function of time, and will be evaluated below in terms of the average plasma energy density. Using the LaPlace transform of $(1)-(3)$, we obtain equations for the current transforms $I_{2}(s)$ and $I_{3}(s)$ :

$$
\begin{aligned}
\left(1+s \tau_{\mathrm{v}}\right) I_{2}+s \tau_{\mathrm{r}} \frac{M_{23}}{L_{22}} I_{3} & =-s \tau_{\mathrm{r}} \frac{M_{12}}{L_{22}} I_{1} \\
s \tau_{L} \frac{M_{23}}{L_{33}} I_{2}+\left(1+s \tau_{L}\right) I_{3} & =-s \tau_{L} \frac{M_{13}}{L_{33}} I_{1}
\end{aligned}
$$

where we have defined the time constants

$$
\begin{gathered}
\tau_{v}=\frac{L_{22}}{R_{2}}=\text { vessel wall time constant } \\
\tau_{L}=\frac{L_{33}}{R_{3}}=\text { diamagneticloop time constant. }
\end{gathered}
$$

Solving (3b) and (4) for $V_{3}(s)$ yields

$$
V_{3}(s)=\frac{1+s \tau_{r} \cdot\left(1-\frac{M_{12} \cdot M_{23}}{L_{22} \cdot M_{13}}\right)}{\left(1+s \tau_{\tau}\right) \cdot\left(1+s \tau_{L}\right)-s^{2} \tau_{t} \tau_{L} k_{23}^{2}} s \cdot M_{13} I_{1}
$$

where

$$
k_{23}^{2}=\frac{M_{23}^{2}}{L_{22} L_{33}}
$$

is the mutual coupling coefficient between the vessel wall and the diamagnetic loop.

When the vessel. plasma, and diamagnetic loop can be modeled as "infinitelylong" cylinders, and the vessel and loop are assumed to have exactly the same radius. evaluation of the inductances under the assumption of perfect magnetic coupling shows that

$$
k_{23}=1, \frac{M_{12} \cdot M_{23}}{L_{22} M_{13}} \approx 1
$$


These approximations are the same as those used by earlier authors (RoTHMAN, 1968; BotTIGLIONI, 1970) where compensation for the low-frequency pole in $V_{3}$ due to flux diffusion through the vessel wall is discussed. With these approximations, (6) reduces to

$$
V_{3}(s)=\frac{1}{1+s\left(\tau_{v}+\tau_{L}\right)} s M_{13} I_{1}
$$

The loop time constant, which in principal can be made very small, simply adds to the vessel time constant to produce a low-frequency s-plane pole in the transfer function. The other pole in (6) disappears to negative infinity as $k_{23}$ approaches unity. The approximations in (8) are for perfect coupling and the resulting transfer function (9) is identical to that of Rothman, modified by the finite time constant of the diamagnetic loop in the denominator of (9). The low-frequency pole caused by finite $\tau_{v}+\tau_{L}$ is easily compensated for by a modified integrator, as shown by Rothman. The finite diamagnetic-loop time constant is included in the present analysis to allow the use of a relatively low loading resistance for cable matching or the reduction of cable ringing.

If imperfect coupling is considered, the approximations of (8) are not satisfied. To address this situation, we define a small parameter $\varepsilon$ where

$$
\varepsilon=1-\frac{M_{12} M_{23}}{L_{22} M_{13}}, 0 \leqslant \varepsilon \leqslant 1
$$

For relatively long, thin plasmas, the magnetic coupling is imperfect, $\varepsilon$ will be small, and $k_{23}$ will be less than unity. Thus, under realistic conditions, (6) has two finite poles and two zeros in the $s$ plane. As $k_{23}$ varies between 0 and 1 , one pole varies between $-\left(\tau_{v}\right)^{-1}\left[\right.$ or $\left.-\left(\tau_{L}\right)^{-1}\right]$ and $-\left(\tau_{v}+\tau_{L}\right)^{-1}$, while the other pole moves from $-\left(\tau_{L}\right)^{-1}\left[\right.$ or $\left.-\left(\tau_{t}\right)^{-1}\right]$ to negative infinity. This pole is referred to as the "highfrequency" pole. The zero in (6) at $-\left(\varepsilon \tau_{v}\right)^{-1}$ tends toward infinity along the negativereal axis. Thus, if $k_{23}$ is not much less than 1 , and $0<\tau_{L} \ll \tau_{r}$. we may factor the denominator into the approximate form

$$
\left(1+s \tau_{r}\right) \cdot\left(1+s \tau_{L}\right)-s^{2} \tau_{r} \tau_{L} k_{23}^{2}=\left[1+s\left(\tau_{i}+\tau_{L}\right)\right] \cdot\left(1+s \tau_{h j}\right)
$$

where $-\left(\tau_{h f}\right)^{-1}$ is the high-frequency pole that is finite when there is less than perfect coupling. For convenience, the low-frequency pole in (6) is retained at $-\left(\tau_{t}+\tau_{L}\right)^{-1}$ in the approximate factoring of (11). With these definitions, (6) becomes

$$
V_{3}(s)=\frac{1+s \tau_{r}^{\varepsilon}}{\left[1+s\left(\tau_{r}+\tau_{L}\right)\right] \cdot\left(1+s \tau_{h f}\right)} s M_{13} I_{1} .
$$

The low-frequency pole at $-\left(\tau_{\mathrm{r}}+\tau_{L}\right)^{-1}$ and the zero at $s=0$ in (12) are compensated for by a modified integrator of the type introduced by Rothman. The values of the high-frequency pole at $-\left(\tau_{h f}\right)^{-1}$ and the high-frequency zero at $-\left(\varepsilon \tau_{t}\right)^{-1}$ can be determined by the calibration process and removed by an active-filter compensation 
circuit as shown in the next section. The transfer function of the compensation circuit is adjusted to cancel all of the poles and zeros of (12).

\section{COMPENSATION AND CALIBRATION CIRCUIT}

An active filter using two operational amplifiers can be constructed to compensate for the frequency dependence implicit in (12). A circuit that accomplishes this for the

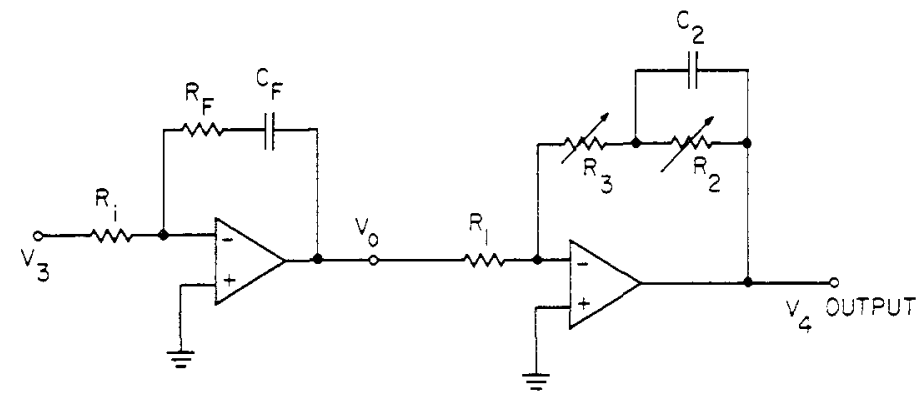

FiG. 2.-Improved compensation circuit for diamagnetic loops located outside the vacuum vessel. This circuit is for the case $\varepsilon t_{t}>i_{h f}$.

case when $\varepsilon \tau_{t}>\tau_{h f}$ is shown in Fig. 2. The first amplifier stage of Fig. 2 cancels the zero at $s=0$ and the low-frequency pole at $-\left(\tau_{r}+\tau_{L}\right)^{-1}$ in $V_{3}(s)$, as shown by Rothman. The second stage cancels the high-frequency pole and zero appearing because of diamagnetic loop loading and imperfect magnetic coupling. Using the transfer function for each stage in Fig. 2, we have

$$
\frac{V_{4}}{I_{1}}=\left(\frac{V_{3}}{I_{1}}\right) \cdot\left(\frac{V_{0}}{V_{3}}\right) \cdot\left(\frac{V_{4}}{V_{0}}\right)
$$

where

$$
\begin{gathered}
\frac{V_{0}}{V_{3}}=-\frac{1+s R_{f} C_{1}}{s R_{i} C_{j}} \cdot \frac{V_{4}}{V_{0}}=-\frac{R_{2}+R_{3}}{R_{1}} \cdot \frac{1+s \tau_{2} f_{2}}{1+s \tau_{2}} \\
\tau_{2}=R_{2} C_{2} \cdot f_{2}=\frac{R_{3}}{R_{2}-R_{3}} .
\end{gathered}
$$

The voltages used in these transfer functions are defined in Fig. 2. The transfer function $V_{3} I_{1}$ is given by (12). Combining these equations gives the overall transfer function

$$
\frac{V_{4}}{I_{1}}=\left(\frac{M_{13}}{R_{i} C_{f}} \cdot \frac{R_{2}+R_{3}}{R_{1}}\right) \cdot\left(\frac{1+s R_{f} C_{f}}{1+s\left(\tau_{i}+\tau_{L}\right)}\right) \cdot\left(\frac{1+s \varepsilon \tau_{f}}{1+s \tau_{h f}}\right) \cdot\left(\frac{1+s \tau_{2} f_{2}}{1+s \tau_{2}}\right) .
$$

Compensation is carried out experimentally by adjusting $R_{f} C_{f} . \tau_{2}$ and $f_{2}$ while 
observing $v_{0}(t)$ or $v_{4}(t)$. The resulting compensation will cancel all poles and zeroes in (13).

A different second-stage circuit is required if $\varepsilon \tau_{v}<\tau_{h f}$. The circuit for this case is given in Fig. 3, and has a transfer function that is the inverse of that for Fig. 2:

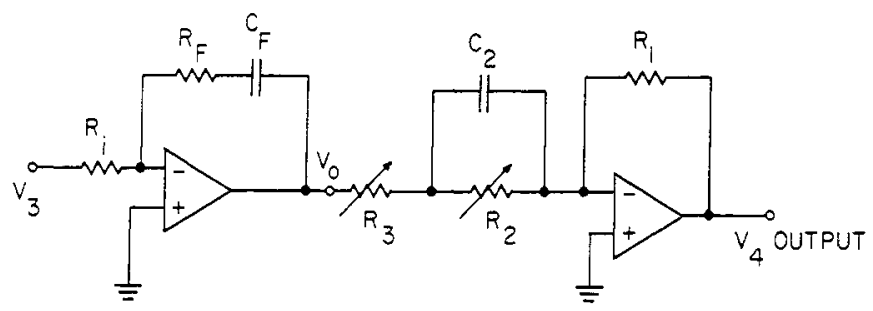

FIG. 3.-Alternative compensation circuit for external diamagnetic loops for the case when

$$
\begin{gathered}
\varepsilon \tau_{\mathrm{r}}<\tau_{h \cdot} . \\
\frac{V_{4}}{V_{0}}=-\frac{R_{1}}{R_{2}+R_{3}} \cdot \frac{1+s \tau_{2}}{1+s \tau_{2} f_{2}} .
\end{gathered}
$$

The selection of the correct circuit can be determined from information obtained in the compensation and calibration procedure.

\section{COMPENSATION AND CALIBRATION PROCEDURE}

The parameters of the compensation circuit can be determined experimentally by using a calibration solenoid to model the plasma diamagnetism. In MIMI, the University of Michigan magnetic-mirror experiment (Booske et al., 1985), a $2.5 \mathrm{~cm}$ diameter, $18 \mathrm{~cm}$ long solenoid was used for compensation and calibration of a 10 $\mathrm{cm}$ diameter diamagnetic loop located concentrically outside the $10 \mathrm{~cm}$ diameter stainless steel vacuum vessel. The solenoid was driven by a rectangular current pulse of approximately $50 \mathrm{~mA}$ amplitude and $400 \mu \mathrm{s}$ duration. The risetime of the current pulse was less than $1 \mu \mathrm{s}$. The solenoid is placed inside the vacuum vessel in approximately the same location occupied by the plasma.

Compensation proceeds by adjusting $R_{f}$ and $C_{f}$ while observing $r_{0}(t)$ so that the equation

$$
R_{f} C_{f}=\tau_{i}+\tau_{L}
$$

is satisfied. The proper values can readily be found by direct observation because this adjustment compensates the d.c. response of $v_{0}(t)$ and the correct signal settles down to a flat top after an initial overshoot or undershoot, as shown in Fig. 4(a and b). If $v_{0}$ overshoots as in Fig. 4(a), then this implies that $\varepsilon \tau_{r}>\tau_{h f}$ and the circuit of Fig. 2 is used for the second stage of compensation. The value of $\tau_{h f}$ can be found from the decay time constant in Fig. $4(a)$, and $\varepsilon \tau_{r}$ is found from the equation

$$
\frac{\varepsilon \tau_{\mathrm{r}}}{\tau_{h f}}=\frac{V_{p}}{V_{\mathrm{ss}}}>1
$$


(a)

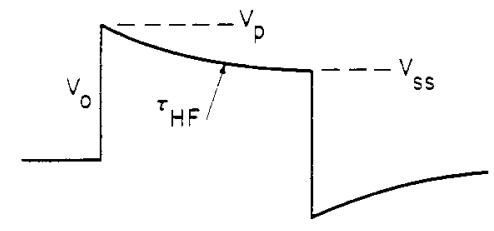

(b)

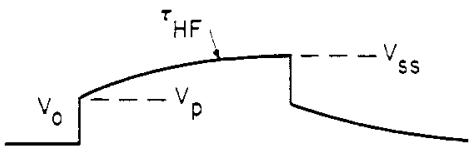

(c)

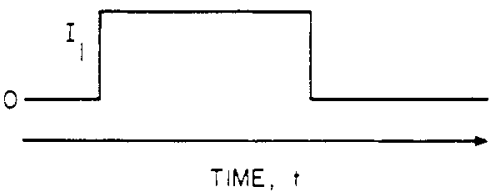

FIG. 4.-Theoretical waveforms for diamagnetic loop compensation. (a) first-stage output voltage $V_{0}(t)$ for $\varepsilon \tau_{h f}:$ (b) first-stage output voltage $V_{0}(t)$ for $\varepsilon \tau_{r}<\tau_{h f}$ and $(c)$ excitation current $I_{1}(t)$.

where $V_{p}$ and $V_{\text {ss }}$ are the peak and steady-state voltages as shown in Fig. 4(a). These relationships can be readily verified by analysing the step response of $v_{0}(t)$ with the low-frequency pole and the zero at $s=0$ removed by the first-stage amplifier of Fig. 2.

If $v_{0}(t)$ undershoots as in Fig. $4(b)$, then $\varepsilon \tau_{r}<\tau_{h f}$ and the circuit of Fig. 3 is used. As in the other case, $\tau_{h f}$ is found from the waveform as the risetime of $v_{0}(t)$ and $\varepsilon \tau_{t}$ is obtained from (15). Once $\varepsilon \tau_{v}$ and $\tau_{h f}$ have been determined. approximate values of $R_{2}$ and $R_{3}$ can be chosen. The pole and zero cancellation requires that $\hat{c} \tau_{r}=\tau_{2}$ and $\tau_{h f}=f_{2} \tau_{2}$ for the case of $\varepsilon \tau_{i}>\tau_{h f}$. or $i \tau_{r}=f_{2} \tau_{2}$ and $\tau_{h f}=\tau_{2}$ if $c \tau_{i}<\tau_{h f}$. The resistors $R_{2}$ and $R_{3}$ are trimmed with potentiometers while observing $t_{4}(t)$ to cancel the remaining high-frequency pole and zero. resulting in the desired frequencyindependent transfer function (for the case $\varepsilon \tau_{r}>\tau_{h j}$ ):

$$
\frac{V_{4}}{I_{1}}=\frac{M_{13}}{R_{i} C_{f}} \cdot \frac{R_{2}+R_{3}}{R_{1}} \triangleq K
$$

The constant $K$ is the proportionally constant between $r_{4}$ and $i_{1}$. The high-frequency limit of (16) is determined by the diffusion time of higher order current modes in the vessel wall and the low-frequency limit will be determined by the active filter operation, i.e. primarily operational amplifier drift.

Using the standard pressure balance equation for a plasma cylinder (see, for example, CHEX, 1974), one can obtain the relationship between the instantaneous average energy density $w(t)$ and the equivalent diamagnetic current $i_{1}(t)$ used in the above analysis. In SI units, this relationship is given for low beta plasmas as 


$$
w \approx n k\left(T_{e}+T_{i}\right) \approx \frac{B_{0} \Delta B}{\mu_{0}} \approx B_{0} h i_{1}
$$

where $B_{0}$ is the axial magnetic field in Tesla and $\Delta B=B_{0}-B$ is the change in $B$ due to plasma diamagnetism. A calibration solenoid with length and diameter chosen to match that of the plasma is excited by a test current $i_{1}$ to produce $\Delta B$. If $\mathrm{h}$ is the number of turns/m of the calibration solenoid, then $\Delta B=\mu_{0} h i_{1}$. Energy density $\mathrm{w}$ is given in terms of the measured voltage $v_{4}$ by

$$
w=\left(\frac{B_{0} h}{K}\right) v_{4} .
$$

The constant $K$ is determined experimentally in the compensation procedure, and can be varied by changing resistor $R_{1}$. After the correct step response is obtained by adjusting the active-filter circuit parameters, $K$ is found by simply taking the ratio of the steady state output voltage $V_{\text {ss }}$ to the input current step $i_{1}$.

\section{RESULTS AND SUMMARY}

This compensation circuit has proven to be very satisfactory in an ECRH experiment where appreciable hot electrons are generated by a $400 \mu \mathrm{s}$ heating pulse. A series of oscilloscope photographs showing the waveforms of $i_{1}, v_{3}, v_{0}$ and $v_{4}$ at the two stages of the compensation procedure are shown in Fig. 5. The driving current $i_{1}$ is a rectangular pulse of $50 \mathrm{~mA}$ magnitude. The values of the various time constants for MIMI were found to be:

$$
\tau_{r}+\tau_{L}=550 \mu \mathrm{s}, \varepsilon \tau_{r}=200 \mu \mathrm{s}, \tau_{h f}=42 \mu \mathrm{s} .
$$

The resulting value of the calibration factor $B_{0} h / K$ was $3.2 \times 10^{13} \mathrm{eV} \mathrm{cm}^{-3} \mathrm{~V}^{-1}$ at $B_{0}=0.264 \mathrm{~T}$. The 1000-turn diamagnetic loop has an axial length of $1.9 \mathrm{~cm}$ and is wound on the outside surface of the vessel at the mirror midplane. The vessel wall thickness is $0.2 \mathrm{~cm}$ and the outside diameter is $10 \mathrm{~cm}$.

The oscilloscope traces shown in Fig. 5 illustrate the waveforms for the driving current $i_{1}(t)$ of the calibration solenoid, raw diamagnetic loop output $v_{3}(t)$, the output $v_{0}(t)$ of the first compensation stage, and the final output $v_{4}(t)$. The first stage of the filter circuit is first adjusted so that $v_{0}$ levels off with a steady state value $V_{s s}$ as shown in Fig. $5(\mathrm{c})$. After this adjustment, the second stage is adjusted to correct the initial overshoot in $v_{0}$, resulting in an optimized response $v_{4}$ as shown in Fig. 5(d). The main remaining error is in the leading edge of $t_{4}$ due to the diffusion of higherorder current modes.

Two additional features were added to the MIMI compensation circuit to remove the effect of ripple and other fluctuations in the confining field magnet current. which is nominally d.c. current. A compensation coil with the same area and turns as the diamagnetic loop was installed in such a way that it links confining field flux but does not link the plasma column or the vessel. The voltage signal from this coil is subtracted from the raw diamagnetic loop voltage signal in a difference amplifier before the signal enters the compensation circuit. In addition, an analog gating circuit is used to gate the compensation circuit input signal. This allows the 


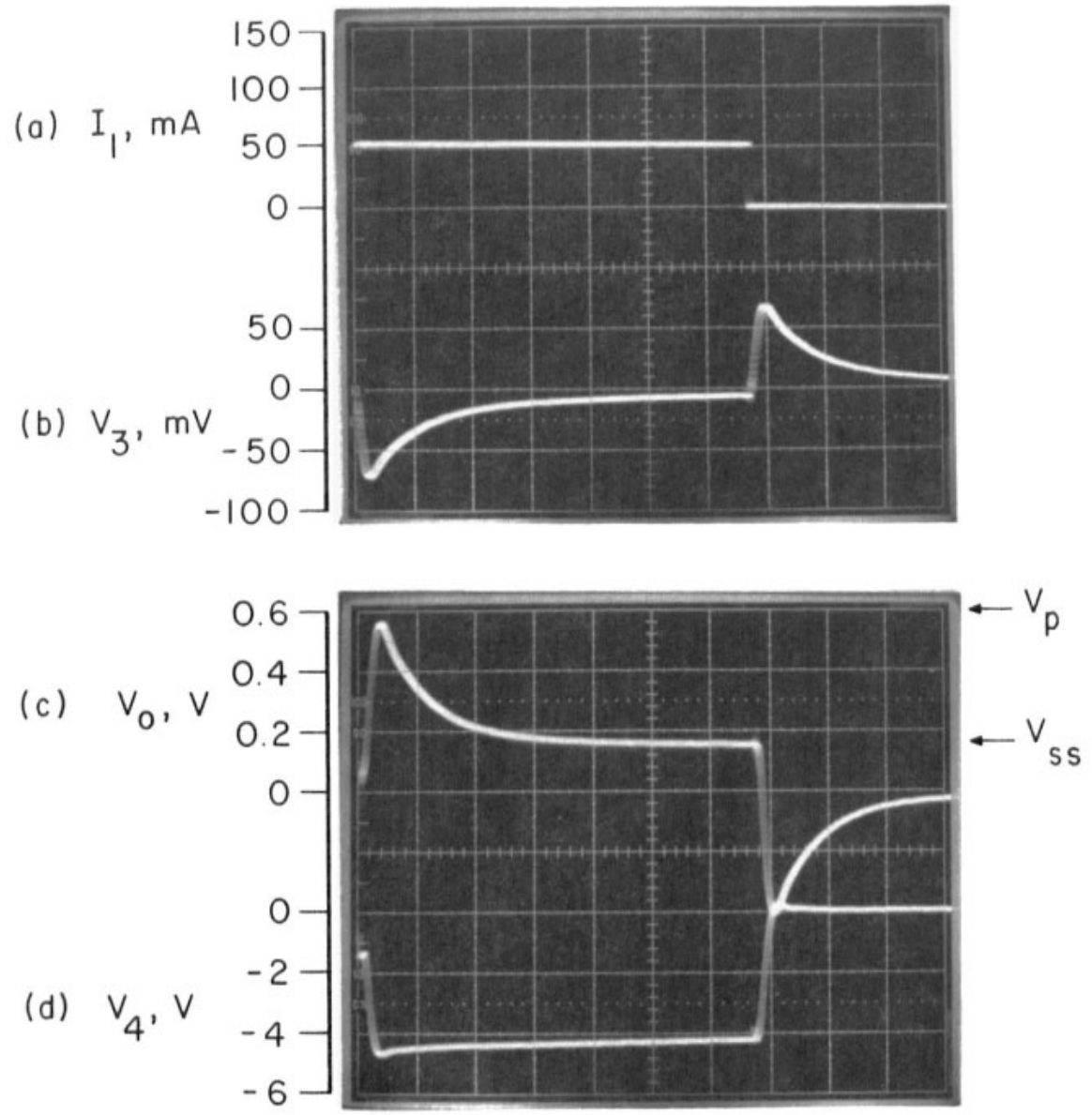

FIG. 5.-Measured calibration waveforms showing the two compensation steps. The recorded quantities are: (a) calibration-solenoid current, $I_{1}$; (b) diamagnetic loop voltage, $V_{3} ;$ (c) first compensation stage voltage, $V_{0}$; and (d) final output voltage, $V_{4} . V_{p}$ and $V_{\text {s }}$ as defined in Fig. 4 are shown on the photographs.

operational amplifier outputs to return to zero between plasma pulses and minimizes the effect of drift. A large resistor is placed in parallel with $C_{f}$ to help reduce the effect of drift in the first stage of the compensation circuit. A high-quality, FETinput, ultra-low-drift operational amplifier is used for this stage. A block diagram of the overall system presently used on MIMI is shown in Fig. 6.

The final result is illustrated by the processed diamagnetic signal from MIMI shown in the oscilloscope trace in Fig. 7. The plasma stored energy builds up slowly due to ECRH and reaches a peak after approximately $200 \mu \mathrm{s}$. At this time diamagnetism falls because an MHD instability causes the density to decrease while the electron conduction loss increases. The ECRH pulse ends at $400 \mu \mathrm{s}$ and at this time the gating circuit opens, causing a small transient in the diamagnetic signal. 


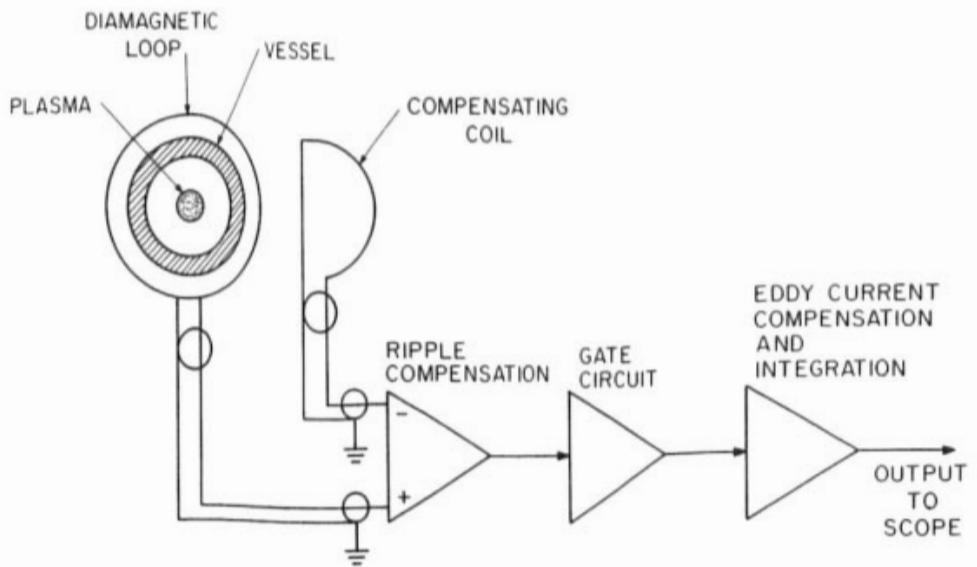

FIG. 6. - Schematic of diamagnetic loop diagnostic with entire signal processing circuit.

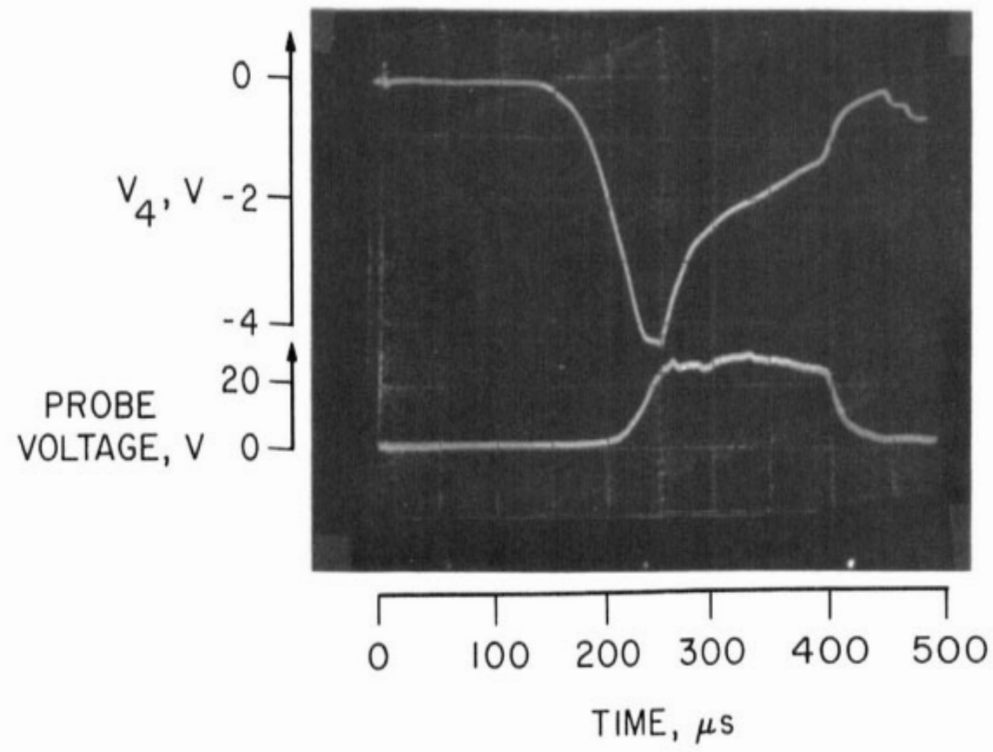

Fig. 7.-A typical plasma diamagnetic signal from MIMI is shown in the upper trace after passing through the ripple-compensation circuit, the gating circuit, and the integrator/vesselcompensation circuit. The lower trace is the Langmuir probe floating potential. The peak of the diamagnetic signal is slightly flattened due to amplifier saturation.

The calibration procedure for the MIMI loop gives a factor of $3.2 \times 10^{13} \mathrm{eV} \mathrm{cm}^{-3}$ $\mathrm{V}^{-1}$ for the operating conditions of Fig. 7 .

Acknowledgements-This work is supported by the National Science Foundation. The support of one of the authors (J.H.B.) by a Magnetic Fusion Energy Technology Fellowship from the Department of Energy is gratefully acknowledged. 


\section{REFERENCES}

Booske J. H., Getry W. D., Gilgenbach R. M. and Jong R. A. (1985) Physics Fluids 28, 3116. BotTiglioni F. (1970) Plasma Physics 12, 131.

CHeN F. F. (1974) Introduction to Plasma Physics, Chapter 6, Plenum Press, New York. Rothmax M. A. (1968) Plasma Physics 10, 86. 